\section{Characteristics of rhegmatogenous retinal detachment and their relationship to visual outcome}

TH Williamson ${ }^{1}$, M Shunmugam ${ }^{1}$, I Rodrigues², M Dogramaci ${ }^{1}$ and E Lee ${ }^{1}$

\begin{abstract}
Aims To examine pre-operative characteristics of rhegmatogenous retinal detachment (RRD) and their relationship to visual acuity (VA) following surgery. Methods Review of prospectively completed electronic database. Baseline characteristics, retinal drawings and outcomes were

following surgery for RRD. Fovea off RRD at presentation achieved poorer postoperative VA than fovea attached and visual outcome was poorer when there was a longer duration of visual symptoms.

Eye (2013) 27, 1063-1069; doi:10.1038/eye.2013.136; published online 21 June 2013
\end{abstract} analysed.

Results In all, 847 eyes from 847 patients with a mean age of 62.2 years, $60 \%$ males, and $56 \%$ right eyes were studied. Mean follow-up was 9.6 months. Preoperative VA correlated with final VA $\left(r^{2}=0.21, P<0.0001\right)$. Median postoperative VA was 6/9 (Logmar 0.18 , quartiles $=0.0-0.48$ ) and was significantly related to anatomical success: $70.15 \%$ achieved 0.18 or better with fully attached retina and primary success, whereas only $8.33 \%$ achieved this when the retina was not fully attached at final follow-up (failure) $(P<0.0001)$. Univariate analysis found multiple variables associated with achieving 0.18 postoperative vision, however, multivariate analysis revealed only primary anatomical success with surgery; absence of proliferative vitreo-retinopathy (PVR), betterpresenting VA and fewer quadrants of detachment were associated with a better visual outcome $\left(r^{2}=0.26, P<0.0001\right)$. Patients with a clinically attached fovea achieved better vision than patients with a clinically detached fovea, independent of the visual loss duration. With foveal detachment however, postoperative VA was better in patients with 1-3 days of visual loss compared with 4-6 days $(P=0.013)$. Conclusions Failure of primary surgery, PVR, extensive RRD and poor-presenting VA are risk factors for poorer visual outcome
Keywords: rhegmatogenous retinal detachment; visual outcome; fovea detachment; retinal detachment surgery

\section{Introduction}

Rhegmatogenous retinal detachment (RRD) is a threat to central vision. With primary anatomical success rates ranging from $81-92 \%$ in uncomplicated cases, ${ }^{1-7} 65-70 \%$ in high-risk eyes, or $75 \%$ when no break is found, ${ }^{2,8-12}$ visual recovery is not certain. Even with successful re-attachment of the retina, the visual acuity (VA) may not return to the pre-morbid level. It would be helpful to the surgeon and patient alike to have an indication as to what preoperative parameters are related to final functional outcome. In addition, identification of controllable factors such as duration of visual symptoms prior to surgery might help plan the appropriate timing of intervention. We have previously used a data set of detailed descriptions of RRD characteristics to show that pseudophakic RRD has a differing pattern of presentation to non-pseudophakic RRD and that it shares similarities to aphakic RRD. ${ }^{13}$ In a larger cohort we have determined, in detail, pre-operative information on patients operated on for RRD and collected VA data at follow-up. In this study, the preoperative characteristics of RRD were studied in relation to visual outcome
${ }^{1}$ Department of Ophthalmology, St Thomas' Hospital, London, UK

${ }^{2}$ Department of Ophthalmology, Queen Mary's Hospital, Sidcup, Kent, UK

Correspondence: TH Williamson, Department of Ophthalmology, St Thomas' Hospital, Lambeth Palace Road, Westminister, London, UK SE1 7EH.

Tel: +44 (0)207 188 4320; Fax: +44 (0)207922 8157. E-mail: Tom@retinasurgery. co.uk

Received: 12 December 2012

Accepted in revised form: 17 May 2013 Published online: 21 June 2013 
to determine if clinical features could identify those achieving good visual recovery.

\section{Materials and methods}

Data from all patients attending a single author's (THW) vitreoretinal surgery service were prospectively entered into an electronic patient record (VITREOR, Microsoft Access, available with the book 'Vitreoretinal Surgery', published by Springer). ${ }^{14}$ This electronic patient record requires recording of retinal detachment characteristics and digital drawings of the RRD, as well enabling the recording of postoperative outcomes; it is therefore a valuable tool for audit and clinical governance. The duration of symptoms at the time of surgery, type of breaks, number of breaks, presence of proliferative vitreo-retinopathy (PVR) according to the revised Retinal Society classification, ${ }^{15}$ extent of retinal detachment, clinical foveal status, presence of vitreous haemorrhage, and VA (determined using Snellen charts, but converted to LogMAR) are recorded. Counting fingers, hand movements and light perception were converted according to Holladay et al. ${ }^{16}$ In addition, preoperative data on the status of the lens (including presence of visually significant cataract or pseudophakia) and date of prior cataract surgery are recorded. Retinal breaks were classified as U-shaped, round (including oval) or mixed shapes. Follow-up required resolution of the gas bubble and was for a minimum of 6 weeks. Ethical approval for the database study was obtained from the local research ethics committee (Guys and St Thomas' Hospitals Trust).

For patients who developed RRD in both eyes, only the first eye was included in the present study. Patients under 40 years of age (to avoid the influence of RRD from young myopes with atrophic holes and vitreous attached), or with aphakia, anterior chamber lens implant, giant retinal tear, retinal dialysis, macular holerelated RRD, retinoschisis-related RRD, and dislocated lens nucleus during cataract surgery were excluded.

Retinal drawings were reviewed by an observer (THW) who was masked to the clinical data of the patient. Drawings were divided into four quadrants centred at the fovea: superotemporal, superonasal, inferotemporal and inferonasal. The observer recorded whether a break was present (true or false) in any of the four quadrants of the eye. This method was used to avoid any conflict of dependent variables that would arise by counting the numbers of breaks in each quadrant and using these as independent variables, ${ }^{17,18}$ which results, in our opinion, in erroneous statistical analysis as multiple breaks in the same eye are not independent variables. The presence of inferior breaks was recorded, specifically noting whether breaks were present between 5 and 7 o'clock (true or false), or at $4-5$ or $7-8$ o' clock (true or false), and whether these were in flat or detached retina. Breaks in these locations can present particular challenges in surgical management, as compared with breaks elsewhere. Eyes were regarded as having an anterior break if a break was identified anterior to a line halfway between the equator and the ora serrata; eyes had posterior break if a break was seen posterior to a line equidistant posteriorly from the equator.

In addition, breaks were regarded as small, medium and large according to their longest meridian and related to the optic disc diameter. A small break was defined as less than half a disc diameter; medium break size was half to two disc diameters, and large more than two disc diameters. Eyes were recorded (true or false) as having small, medium or large breaks. Thus, a single eye could have small, medium and large breaks if all sizes were present.

Success was defined in three categories at final followup:

1. Primary success with a fully attached retina without intraocular tamponade with one planned procedure (excluding oil removal) or two procedures if a planned delayed retinectomy was performed for PVR. ${ }^{19}$

2. Secondary success when a fully flat retina even with silicone oil in situ was achieved outside those parameters described in 1.

3. Final failure with any area of retinal detachment at final follow-up.

For most of the statistical comparisons, category 1 (primary success) have been compared to a combination of both 2 and 3 (primary failure). The primary outcome measure was the achievement of 6/9 vision (LogMAR 0.18 ) or better. To determine the effect of duration of visual loss on visual recovery for patients with and without evidence of a clinically detached fovea (fovea off), patients with primary success of surgery and no PVR were further analysed. Data were analysed using statistical software for univariate analysis (Analyse-it, Leeds, UK) and multivariate analysis (Statplus, Analystsoft, Vancouver, BC, Canada). Means were compared using the $t$-test, medians by nonparametric methods and proportions using Fisher's exact test or Pearson $\chi^{2}$-test.

\section{Results}

In all, 847 eyes from 847 patients were included in the study, and baseline characteristics are shown in Table 1. Mean age was 62.2 years (range 40-97 years). Mean follow-up was 9.6 months. Median number of breaks 
was 2, with mean preoperative VA LogMAR 0.93, SD 0.86 (6/51 Snellen equivalent). Mean spherical equivalent was - 1.77D (range - 18.25-4D).

Median postoperative vision was LogMAR 0.18 (quartiles $0.0-0.48$ ) or 6/9 Snellen equivalent. Proportions of patients achieving different levels of VA were $27.1 \%$ achieved $6 / 6$ or better, $39.0 \% 6 / 9$ to $6 / 12$, $24.6 \% 6 / 15$ to $6 / 60,7.4 \% 6 / 120$ to counting fingers, $1.8 \%$ hand movements, and $0.1 \%$ light perception (Figure 1). Final vision was significantly related to the success of surgery with primary success achieving $6 / 9$ or better in $70.15 \%$, secondary success $47.67 \%$ and failure $8.33 \%$, $P<0.0001$.

Table 1 Baseline characteristics

\begin{tabular}{lc}
\hline Patient characteristics & $\mathrm{n}(\%)$ \\
\hline Sex & \\
Female & $338(39.9)$ \\
Male & $509(60.1)$ \\
& \\
Eye & \\
Right & $471(55.6)$ \\
Left & $376(44.4)$ \\
& \\
Lens & \\
Normal crystalline lens & $329(38.8)$ \\
Posterior chamber IOL & $253(29.9)$ \\
Cortical cataract & $49(5.8)$ \\
Mixed cataract & $22(2.6)$ \\
Nuclear sclerotic cataract & $179(21.1)$ \\
Posterior subcapsular cataract & $15(1.8)$ \\
& \\
Detachment characteristics & \\
Fovea-off & \\
Quadrants of RRD & $477(56.3)$ \\
1 & \\
2 & $175(24.4)$ \\
3 & $301(35.5)$ \\
4 & $228(26.9)$ \\
PVR (grades B or C) & $112(13.2)$ \\
\hline
\end{tabular}

Using univariate analysis, the following variables were associated with worse postoperative vision: presence of PVR; vitrectomy surgery; presence of U-tears; breaks in RRD between 5-7 clock hours; increased quadrants of RRD; fovea off; breaks inferotemporally; presence of preoperative cataract; increasing age; worse VA at presentation, and increased duration of follow-up (Table 2 and Supplementary Data Table). Preoperative VA was correlated with final VA $r^{2}=0.21, P<0.0001$ (Figure 2). After multivariate analysis, primary success of surgery, PVR, presenting VA and number of quadrants of RRD were significantly associated with the visual outcome, $r^{2}=0.26, P<0.0001$ (Table 2 ).

\section{Analysis of foveal status and duration of visual loss}

To assess the effect detachment of the fovea and the duration of visual loss had on visual outcomes, only those with primary success and no PVR were examined (330 patients with fovea on and 325 patients with fovea off). There were missing data for duration of visual loss for 25 patients with fovea off RRD. By univariate analysis, increased follow-up duration, U-tears, mixed breaks, increased quadrants of RRD, fovea off, breaks in detached superotemporal retina, worse VA at presentation, and increased duration of vision loss were related to worse final VA. After multivariate analysis presenting vision and duration of vision loss were related to final VA, $r^{2} 0.15, P<0.0001$.

Patients with fovea attached achieved significantly better vision (median 6/6) than patients with fovea off (median 6/9) independent of the duration of visual loss (1, 2, 3, 4, 5, 6, 7-13, 14-20, or $\geq 21$ days), Figure 3. Once the fovea was detached, visions were similar with 1, 2 and 3 days of visual loss, but were significantly better at $1-3$ days (median 0.18 , quartiles $0.0,0.48$ ) compared with 4-6 days (median 0.30, quartiles 0.18, 0.60, $P=0.013$ ); there was no difference between groups thereafter.

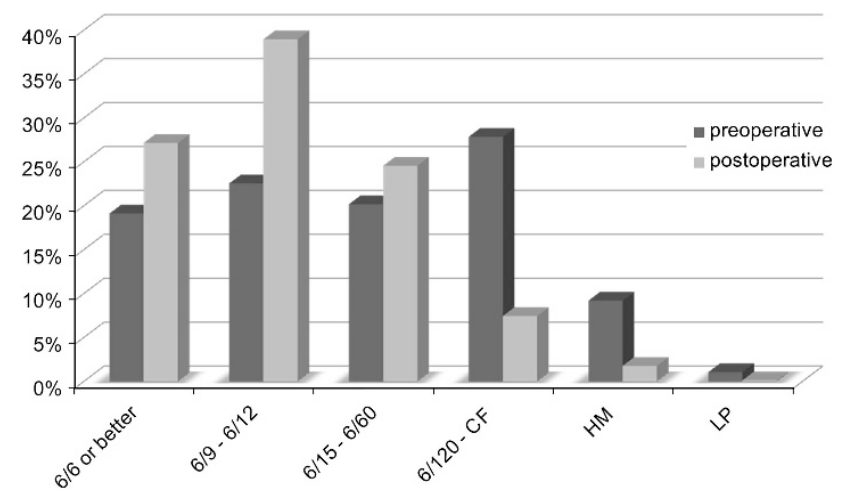

Figure 1 Proportions of patients with levels of Snellen acuity preoperatively and postoperatively, $\mathrm{CF}=$ counting fingers, $\mathrm{HM}=$ hand movements, $\mathrm{LP}=$ light perception. 
Table 2 The proportion of patients with 6/9 vision or better at follow-up and the relationship with variables that were significantly related to follow-up vision after multivariate analysis

\begin{tabular}{|c|c|c|c|c|c|}
\hline & $\begin{array}{c}6 / 9 \text { or better vision } \\
\text { with variable }(\%)\end{array}$ & $\begin{array}{c}6 / 9 \text { or better vision } \\
\text { without variable (\%) }\end{array}$ & $\begin{array}{l}\text { Relative } \\
\text { risk }\end{array}$ & $\begin{array}{l}95 \% \text { confidence } \\
\text { interval }\end{array}$ & P-value \\
\hline Primary success of surgery & 70.15 & 39.09 & 2.04 & $1.69-2.46$ & $<0.0001$ \\
\hline Presence of PVR & 32.50 & 71.66 & 0.42 & $0.35-0.50$ & $<0.0001$ \\
\hline Quadrants of RRD 3 or 4 & 47.94 & 78.30 & 0.42 & $0.32-0.51$ & $<0.0001$ \\
\hline Visual acuity at presentation of $20 / 30$ or better & 87.25 & 51.01 & 3.84 & $2.88-5.12$ & $<0.0001$ \\
\hline
\end{tabular}

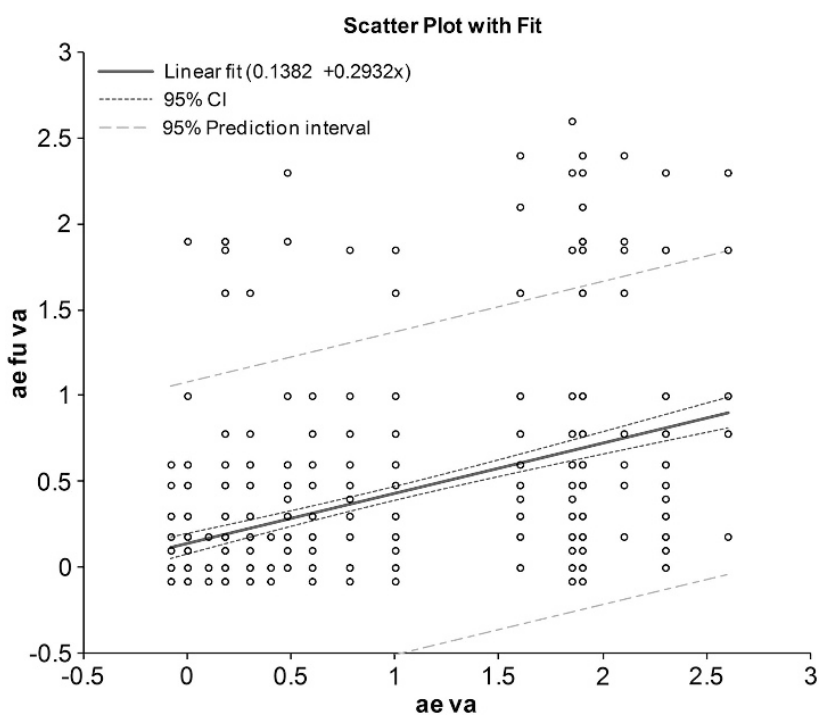

Figure 2 Relationship of preoperative VA (ae va) to postoperative acuity (ae fu va), in LogMAR units. The correlation between preoperative and postoperative acuity is significant with $P<0.0001$ and $r^{2}=0.21$.

\section{Discussion}

Visual recovery after surgery for RRD shows that high-to-moderate levels of VA can be achieved in most patients in this series with $27.1 \%$ achieving $6 / 6$ or better, $39.0 \% 6 / 9$ to $6 / 12$, and a further $24.6 \% 6 / 15$ to $6 / 60$. Primary success of surgery, absence of PVR, betterpresenting VA and fewer of quadrants of RRD were significantly associated with achieving vision of $6 / 9$ or better when all patients were included. This is similar to other studies. ${ }^{20,21}$ Preoperative VA also correlated with final visual outcomes; the better the preoperative acuity the better the postoperative acuity. Similarly those patients whose retina was successfully re-attached with one procedure experienced better functional outcomes. PVR is the strongest risk factor for failure of surgery and therefore it is unsurprising that it was associated with reduced vision.

The relationship of poorer vision with increased involvement of quadrants of RRD is of interest and has been noted before. ${ }^{20}$ The effect of increased subretinal fluid volume on poorer recovery of the retina has been suggested before by the comparison of good recovery of vision with central serous chorioretinopathy, in comparison to poorer recover with RRD.

The examination of patients with primary success and no PVR allowed a best-case scenario examination of visual expectation, and in particular allowed an investigation of foveal detachment and duration of visual loss. Having an attached fovea allowed a better visual outcome compared to fovea detached, even if the symptoms of visual loss were of 1 day only. The visual loss is limited to deterioration from median $6 / 6$ vision in fovea on patients to median $6 / 9$ in fovea off patients, but there may be other symptoms such as distortion and micropsia, which are more prevalent once the fovea detaches. ${ }^{22}$ Although other published studies have not found an effect on anatomical or functional outcomes following delays in surgery for macula on retinal detachments, very few if any of their patients became macular off while waiting for surgery. ${ }^{23-25}$ The results presented here demonstrate that there is a worse outcome following foveal involvement and therefore, as the rate of detachment cannot be accurately predicted, 


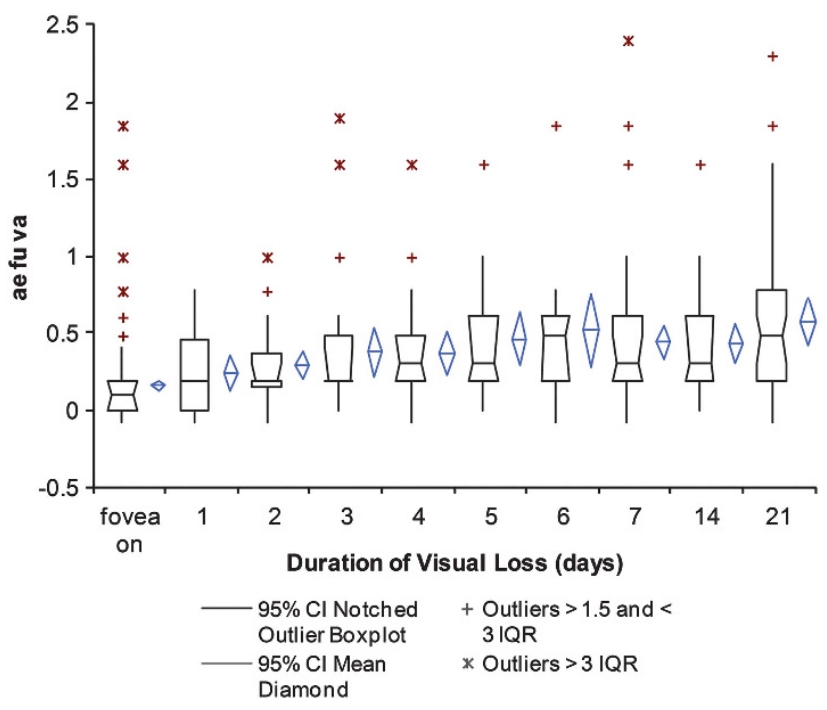

Figure 3 Visual acuities after surgery (ae fu va) in fovea on patients and fovea off patients with 1, 2, 3, 4, 5, 6, 7-13 (7), 14-20 (14), or $\geq 21$ days (21) duration of visual loss.

surgeons need to perform prompt surgery for fovea on detachments to avoid progression and foveal detachment. $^{26}$

Surgeons have been reassured by publications that show no difference in visual recovery in patients with foveal off RRD and varying duration of symptoms in the first week ${ }^{27-31}$ or first 10 days. ${ }^{32}$ This has lead to Ross et $a l^{29}$ suggesting that macular off RRD can wait for a scheduled operating list. However, our study did not demonstrate findings in agreement with this, as patients operated within 1-3 days of symptoms did better than those with a longer duration of symptoms. This may have been due to the removal of patients with PVR, which would be expected to occur later and be associated with poorer surgical success and visual results. Inclusion of patients with PVR may explain the results of previous studies in this area that have shown continued deterioration of visual recovery weeks later. ${ }^{33-35}$

A potential flaw of the present study is the definition of visual loss, which could vary from peripheral visual field loss to central visual loss. As the former could occur prior to central loss, there may be patients recorded as having long durations of visual loss, who actually had only short durations of central loss, and therefore achieved better visual outcomes than would be expected. In addition, operation by PPV induces cataract, which reduces visual results and confounds interpretation of visual results in any study of PPV. After multivariate analysis, crystalline lens, pseudophakia and preoperative cataract were not associated with further visual loss, perhaps because any cataract was readily dealt with by peroperative or postoperative cataract surgery.
In conclusion, visual recovery after surgery for RRD is usually high to moderate. PVR, failure of surgery, extensive RRD, and poor-presenting VA are risk factors for a poorer visual outcome. Eyes with fovea off RRD at presentation achieved less visual recovery than patients with fovea attached, while a duration of visual symptoms of $<3$ days achieved better vision than longer durations in patients with foveal detachment.

\section{Summary}

What was known before

- Preoperative characteristics that affect anatomical success of surgery for rhegmatogenous retinal detachment are well known, but factors influencing visual outcomes are not.

- Visual recovery following surgery for rhegmatogenous retinal detachment (RRD) is variable and unpredictable.

What this study adds

- Visual outcomes following surgery for RRD are generally good. PVR, failure of surgery, extensive RRD, and poorpresenting VA are risk factors for a poorer visual outcome.

- Foveal detachment pre-operatively had less visual recovery than patients with fovea attached, while duration of visual symptoms $<3$ days achieved better vision than longer durations in patients with foveal detachment.

- Consideration of these factors is important when counselling patients of the likely visual prognosis and when determining the urgency of surgical intervention. 


\section{Conflict of interest}

The senior author (THW) has commercial interests in VITREOR, the electronic database used in this study. All other authors declare no conflict of interest.

\section{Acknowledgements}

This study was funded by Eyehope (UK registered charity 1119866).

\section{Disclaimer}

The funding organisation had no role in the design or conduct of this research.

\section{References}

1 Ah-Fat FG, Sharma MC, Majid MA, McGalliard JN, Wong D. Trends in vitreoretinal surgery at a tertiary referral centre: 1987 to 1996. Br J Ophthalmol 1999; 83(4): 396-398.

2 Campo RV, Sipperley JO, Sneed SR, Park DW, Dugel PU, Jacobsen J et al. Pars plana vitrectomy without scleral buckle for pseudophakic retinal detachments. Ophthalmology 1999; 106(9): 1811-1815.

3 Girard P, Karpouzas I. Pseudophakic retinal detachment: anatomic and visual results. Graefes Arch Clin Exp Ophthalmol 1995; 233(6): 324-330.

4 La Heij EC, Derhaag PF, Hendrikse F. Results of scleral buckling operations in primary rhegmatogenous retinal detachment. Doc Ophthalmol 2000; 100(1): 17-25.

5 Oshima Y, Emi K, Motokura M, Yamanishi S. Survey of surgical indications and results of primary pars plana vitrectomy for rhegmatogenous retinal detachments. Jpn J Ophthalmol 1999; 43(2): 120-126.

6 Thompson JA, Snead MP, Billington BM, Barrie T, Thompson JR, Sparrow JM. National audit of the outcome of primary surgery for rhegmatogenous retinal detachment. II. Clinical outcomes. Eye 2002; 16(6): 771-777.

7 Minihan M, Tanner V, Williamson TH. Primary rhegmatogenous retinal detachment: 20 years of change. Br J Ophthalmol 2001; 85(5): 546-548.

8 Hakin KN, Lavin MJ, Leaver PK. Primary vitrectomy for rhegmatogenous retinal detachment. Graefes Arch Clin Exp Ophthalmol 1993; 231(6): 344-346.

9 Heimann H, Bornfeld N, Friedrichs W, Helbig H, Kellner U, Korra A et al. Primary vitrectomy without scleral buckling for rhegmatogenous retinal detachment. Graefes Arch Clin Exp Ophthalmol 1996; 234(9): 561-568.

10 Schmidt JC, Rodrigues EB, Hoerle S, Meyer CH, Kroll P. Primary vitrectomy in complicated rhegmatogenous retinal detachment-a survey of 205 eyes. Ophthalmologica 2003; 217(6): 387-392.

11 Tewari HK, Kedar S, Kumar A, Garg SP, Verma LK. Comparison of scleral buckling with combined scleral buckling and pars plana vitrectomy in the management of rhegmatogenous retinal detachment with unseen retinal breaks. Clin Experiment Ophthalmol 2003; 31(5): 403-407.
12 Wong D, Billington BM, Chignell AH. Pars plana vitrectomy for retinal detachment with unseen retinal holes. Graefes Arch Clin Exp Ophthalmol 1987; 225(4): 269-271.

13 Mahroo OA, Dybowski R, Wong R, Williamson TH. Characteristics of rhegmatogenous retinal detachment in pseudophakic and phakic eyes. Eye (Lond) 2012; 26(8): 1114-1121.

14 Williamson TH. Vitreoretinal Surgery. Springer: Berlin, Germany, 2008.

15 Machemer R, Aaberg TM, Freeman HM, Irvine AR, Lean JS, Michels RM. An updated classification of retinal detachment with proliferative vitreoretinopathy. Am J Ophthalmol 1991; 112(2): 159-165.

16 Holladay JT. Proper method for calculating average visual acuity. J Refract Surg 1997; 13(4): 388-391.

17 Phillips CI. Distribution of breaks in aphakic and "senile" eyes with retinal detachments. Br J Ophthalmol 1963; 47: 744-752.

18 Ashrafzadeh MT, Schepens CL, Elzeneiny II, Moura R, Morse P, Kraushar MF. Aphakic and phakic retinal detachment. I. Preoperative findings. Arch Ophthalmol 1973; 89(6): 476-483.

19 Williamson TH, Gupta B. Planned delayed relaxing retinotomy for proliferative vitreoretinopathy. Ophthalmic Surg Lasers Imaging 2010; 41(1): 31-34.

20 Salicone A, Smiddy WE, Venkatraman A, Feuer W. Visual recovery after scleral buckling procedure for retinal detachment. Ophthalmology 2006; 113(10): 1734-1742.

21 Oshima Y, Yamanishi S, Sawa M, Motokura M, Harino S, Emi K. Two-year follow-up study comparing primary vitrectomy with scleral buckling for macula-off rhegmatogenous retinal detachment. Jpn J Ophthalmol 2000; 44(5): 538-549.

22 Ugarte M, Williamson TH. Horizontal and vertical micropsia following macula-off rhegmatogenous retinaldetachment surgical repair. Graefes Arch Clin Exp Ophthalmol 2006; 244(11): 1545-1548.

23 Ehrlich R, Niederer RL, Ahmad N, Polkinghorne P. Timing of acute macula-on rhegmatogenous retinal detachment repair. Retina 2013; 33(1): 105-110.

24 Wykoff CC, Smiddy WE, Mathen T, Schwartz SG, Flynn Jr, HW, Shi W. Fovea-sparing retinal detachments: time to surgery and visual outcomes. Am J Ophthalmol 2010; 150(2): 205-210.

25 Lai MM, Khan N, Weichel ED, Berinstein DM. Anatomic and visual outcomes in early versus late macula-on primary retinal detachment repair. Retina 2011; 31(1): 93-98.

26 Carifi G. Disagreement with some statements from Lai et al. Retina 2011; 31(8): 1744-1745.

27 Ross W, Lavina A, Russell M, Maberley D. The correlation between height of macular detachment and visual outcome in macula-off retinal detachments of $<$ or $=7$ days' duration. Ophthalmology 2005; 112(7): 1213-1217.

28 Ross WH. Visual recovery after macula-off retinal detachment. Eye 2002; 16(4): 440-446.

29 Ross WH, Kozy DW. Visual recovery in macula-off rhegmatogenous retinal detachments. Ophthalmology 1998; 105(11): 2149-2153.

30 Ross WH, Stockl FA. Visual recovery after retinal detachment. Curr Opin Ophthalmol 2000; 11(3): 191-194.

31 Diederen RM, La Heij EC, Kessels AG, Goezinne F, Liem AT, Hendrikse F. Scleral buckling surgery after 
macula-off retinal detachment: worse visual outcome after more than 6 days. Ophthalmology 2007; 114(4): 705-709.

32 Hassan TS, Sarrafizadeh R, Ruby AJ, Garretson BR, Kuczynski B, Williams GA. The effect of duration of macular detachment on results after the scleral buckle repair of primary, macula-off retinal detachments. Ophthalmology 2002; 109(1): 146-152.

33 Abouzeid H, Wolfensberger TJ. Macular recovery after retinal detachment. Acta Ophthalmol Scand 2006; 84(5): 597-605.
34 Wolfensberger TJ, Gonvers M. Optical coherence tomography in the evaluation of incomplete visual acuity recovery after macula-off retinal detachments. Graefes Arch Clin Exp Ophthalmol 2002; 240(2): 85-89.

35 Yang CH, Lin HY, Huang JS, Ho TC, Lin CP, Chen MS et al. Visual outcome in primary macula-off rhegmatogenous retinal detachment treated with scleral buckling. J Formos Med Assoc 2004; 103(3): 212-217.

Supplementary Information accompanies this paper on Eye website (http://www.nature.com/eye) 